\title{
WU-CRISPR: characteristics of functional guide RNAs for the CRISPR/Cas9 system

\author{
Nathan Wong ${ }^{1,2}$, Weijun Liu $^{2}$ and Xiaowei Wang ${ }^{2, *^{*}}$
}

\begin{abstract}
The CRISPR/Cas9 system has been rapidly adopted for genome editing. However, one major issue with this system is the lack of robust bioinformatics tools for design of single guide RNA (sgRNA), which determines the efficacy and specificity of genome editing. To address this pressing need, we analyze CRISPR RNA-seq data and identify many novel features that are characteristic of highly potent sgRNAs. These features are used to develop a bioinformatics tool for genome-wide design of sgRNAs with improved efficiency. These sgRNAs as well as the design tool are freely accessible via a web server, WU-CRISPR (http://crispr.wustl.edu).
\end{abstract}

\section{Background}

The CRISPR/Cas9 system has been developed in recent years for genome editing, and it has been rapidly and widely adopted by the scientific community [1]. The RNA-guided enzyme Cas9 originates from the CRISPRCas adaptive bacterial immune system. CRISPRs (clustered regularly interspaced palindromic repeats) are short repeats interspaced with short sequences in bacteria genomes. CRISPR-encoded RNAs have been shown to serve as guides for the Cas protein complex to defend against viral infection or other types of horizontal gene transfer by cleaving foreign DNA [2-4]. Major progress has been made recently to modify the natural CRISPR/ Cas9 process in bacteria for applications in mammalian genome editing $[5,6]$. Compared with other genome editing methods, the CRISPR system is simpler and more efficient, and can be readily applied to a variety of experimental systems [7-11].

The natural CRISPR/Cas9 system in bacteria has two essential RNA components, mature CRISPR RNA (crRNA) and trans-activating crRNA (tracrRNA). These two RNAs have partial sequence complementarity and together form a well-defined two-RNA structure that directs Cas9 to target invading viral or plasmid DNA [2, 12]. Recent work indicates that it is feasible to engineer a single RNA chimera (single guide RNA, or sgRNA) by combining the

\footnotetext{
* Correspondence: xwang@radonc.wustl.edu

${ }^{2}$ Department of Radiation Oncology, Washington University School of Medicine, St Louis, MO 63108, USA

'Department of Biomedical Engineering, Washington University, St Louis, MO 63130, USA
}

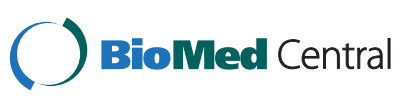

(c) 2015 Wong et al. Open Access This article is distributed under the terms of the Creative Commons Attribution 4.0 International License (http://creativecommons.org/licenses/by/4.0/), which permits unrestricted use, distribution, and reproduction in any medium, provided you give appropriate credit to the original author(s) and the source, provide a link to the Creative Commons license, and indicate if changes were made. The Creative Commons Public Domain Dedication waiver (http://creativecommons.org/publicdomain/zero/1.0/) applies to the data made available in this article, unless otherwise stated.

sequences of both crRNA and tracrRNA [13]. The sgRNA is functionally equivalent to the crRNA-tracrRNA complex, but is much simpler as a research tool for mammalian genome editing. In a typical CRISPR study, an sgRNA is designed to have a guide sequence domain (designated as gRNA in our study) at the $5^{\prime}$ end, which is complementary to the target sequence. The rationally designed sgRNA is then used to guide the Cas9 protein to specific sites in the genome for targeted cleavage.

The gRNA domain of the sgRNA determines both the efficacy and specificity of the genome editing activities by Cas9. Given the critical roles of gRNA, multiple bioinformatics tools have been developed for rational design of gRNAs for the CRISPR/Cas9 system [14-17]. Experimental analysis indicates that Cas9-based genome editing could have widespread off-target effects, resulting in a significant level of non-specific editing at other unintended genomic loci $[14,18-20]$. Thus, most existing design tools have focused primarily on selection of gRNAs with improved specificity for genome targeting. However, more recent studies have demonstrated that the off-target effects of the CRISPR-Cas9 system is not as extensive as previously speculated, and random targeting of the noncoding regions in the genome has little functional consequences in general [21, 22]. Furthermore, novel experimental systems have been developed to improve the targeting specificity of CRISPR/Cas9 [23, 24]. Besides targeting specificity, another important aspect of bioinformatics design is to select gRNAs with high targeting potency. Individual gRNAs vary greatly in their efficacy to guide Cas9 for genome editing. Thus, the 
design of potent gRNAs is highly desired, as inefficient genome editing by Cas9 will inevitably lead to significant waste of resources at the experimental screening stage. The importance of gRNA efficacy has only been appreciated very recently, with multiple studies attempting to identify sequence features that are relevant to functionally active sgRNAs [21, 25-28]. For example, one recent study by Doench and colleagues [21] analyzed 1841 randomly selected gRNAs and identified position-specific sequence features that are predictive of gRNA potency. Similarly, CRISPRseek is a BioConductor package that also implements the Doench algorithm for potency prediction [29]. In our study, we reanalyzed this public dataset and identified many novel features that are characteristic of functional gRNAs. These selected features have been integrated into a bioinformatics algorithm for the design of gRNAs with high efficacy and specificity. A web server implementing this design algorithm has also been established.

\section{Results}

In a recent study, Doench and colleagues [21] analyzed $1841 \mathrm{sgRNAs}$ to identify sequence features that are associated with CRISPR activities. From that analysis, significant position-specific sequence features have been discovered. In particular, nucleotides adjacent to the protospacer adjacent motif (PAM) NGG in the target site are significantly depleted of $\mathrm{C}$ or $\mathrm{T}$. In our study, this public dataset was systematically reanalyzed to identify other novel features that are predictive of CRISPR activity. To this end, we compared the most potent sgRNAs (top $20 \%$ in ranking) with the least potent sgRNAs (bottom $20 \%$ ). By excluding sgRNAs with modest activities in this manner, distinct characteristics of functional sgRNAs can be more readily identified. The same strategy for feature selection has been proven to be effective in our previous study to characterize highly active small interfering RNAs (siRNAs) for target knockdown [30].

\section{Structural characteristics of functional sgRNAs}

Previous studies have shown that structural accessibility plays an important role in RNA-guided target sequence recognition, such as by siRNA and microRNA [30-32]. Similarly, we hypothesized that structural characteristics of the sgRNA are important determinants of CRISPR activity. To this end, RNA secondary structures were calculated with RNAfold [33]. Overall secondary structure, self-folding free energy, and the accessibility of individual nucleotides in the structure were analyzed for each sgRNA. The sgRNA consists of two functional domains, the gRNA sequence and tracrRNA sequence. The gRNA sequence consists of 20 nucleotides that pair perfectly to the targeted genomic sequence, thereby guiding the recruitment of the Cas9 protein to the target site; on the other hand, tracrRNA binds to Cas9 to form a functionally active RNA-protein complex. As shown in Fig. 1a, the tracrRNA region contains multiple well-defined structural motifs, which are important for interaction with Cas9 to form a functional RNA-protein complex.

Compared with non-functional sgRNAs, functional sgRNAs were significantly more accessible at certain nucleotide positions (Fig. 1b, c). In particular, the most significant difference in accessibility involved nucleotides at positions $18-20$, which constitute the $3^{\prime}$ end of the guide sequence (highlighted in Fig. 1a). The 3' end of the guide sequence, also known as the "seed region", plays a critical role in recognition of target sequence. Thus, based on structural analysis, accessibility of the last three bases in the seed region was a prominent feature to differentiate functional sgRNAs from non-functional ones (Fig. 1b). In addition, base accessibility in positions $51-53$ was also significantly different. In the predicted structure of the sgRNA, nucleotides at positions 21-50 form a stable stem-loop secondary structure. From the survey of non-functional sgRNAs, nucleotides at positions 51-53 commonly paired with the end nucleotides of the guide sequence (positions 18-20), resulting in an extended stem-loop structure encompassing positions 18-53. Thus, decreased base accessibility at positions 51-53 was generally associated with decreased accessibility of the end of the seed region.

Furthermore, overall structural stability of the guide sequence alone (i.e., the gRNA domain comprising positions 1-20) was evaluated with thermodynamics analysis. Specifically, the propensity to form secondary structure was determined by calculating the self-folding free energy of the guide sequence. On average, non-functional guide sequences had significantly higher potential for self-folding than functional ones, with $\Delta \mathrm{G}=-3.1$ and -1.9 , respectively $(P=6.7 \mathrm{E}-11$; Fig. $2 \mathrm{a})$. Thus, the result from thermodynamic analysis also indicated that structural accessibility of the guide sequence was correlated with sgRNA functionality. In general, structural stability of the RNA can be approximated by the GC content of the sequence. Consistent with the free energy calculation, the guide sequence of non-functional sgRNAs had higher GC content on average compared with functional sgRNAs ( 0.61 versus $0.57, P=2.1 \mathrm{E}-5)$. Furthermore, thermodynamic stability of the gRNA/target sequence was evaluated. On average, non-functional guide sequences were predicted to form more stable RNA/DNA duplexes with the target sequence than functional ones, with $\Delta G=-17.2$ and -15.7 , respectively $(P=4.9 \mathrm{E}-10$; Fig. $2 \mathrm{~b})$. Thus, high duplex stability was a significant characteristic of non-functional sgRNAs.

\section{Sequence characteristics of functional sgRNAs}

In addition to structural features describing the sgRNA, relevant sequence features of the guide sequence were also evaluated and are presented below. 

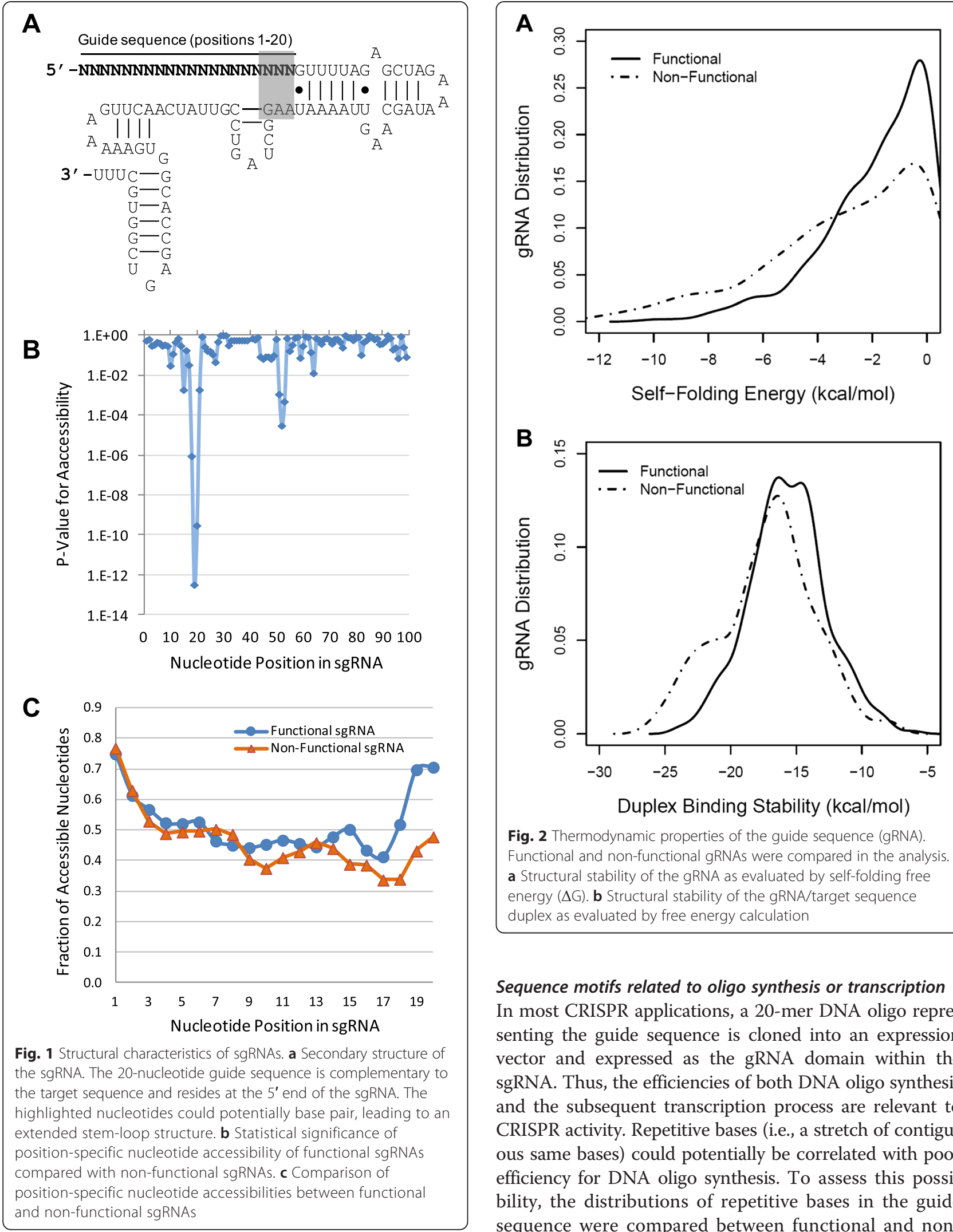

Fig. 2 Thermodynamic properties of the guide sequence (gRNA). Functional and non-functional gRNAs were compared in the analysis. a Structural stability of the gRNA as evaluated by self-folding free energy $(\Delta \mathrm{G})$. $\mathbf{b}$ Structural stability of the gRNA/target sequence duplex as evaluated by free energy calculation

\section{Sequence motifs related to oligo synthesis or transcription} In most CRISPR applications, a 20-mer DNA oligo representing the guide sequence is cloned into an expression vector and expressed as the gRNA domain within the sgRNA. Thus, the efficiencies of both DNA oligo synthesis and the subsequent transcription process are relevant to CRISPR activity. Repetitive bases (i.e., a stretch of contiguous same bases) could potentially be correlated with poor efficiency for DNA oligo synthesis. To assess this possibility, the distributions of repetitive bases in the guide sequence were compared between functional and nonfunctional gRNAs. Repetitive bases are defined as any of the following: five contiguous adenines, five contiguous 
cytosines, four contiguous guanines, or four contiguous uracils. Overall, compared with non-functional gRNAs, functional gRNAs were significantly depleted of repetitive bases ( $5.4 \%$ versus $22.8 \%, P=1.3 \mathrm{E}-11$ ). Among the four bases, four contiguous guanines (GGGG) were especially correlated with poor CRISPR activity. Previous work indicates that GGGG not only leads to poor yield for oligo synthesis, but also has the propensity to form a special secondary structure called a guanine tetrad, which makes the guide sequence less accessible for target sequence recognition. Consistently, much fewer functional gRNAs were observed to contain the GGGG motif than nonfunctional ones ( $4.9 \%$ versus $17.9 \%, P=2.6 \mathrm{E}-8)$.

A stretch of contiguous uracils signals the end of transcription for RNA polymerase III, which recognizes the U6 promoter. All gRNAs containing UUUU in the guide sequence had been preselected for exclusion from our analysis. Furthermore, recent work suggested that three repetitive uracils (UUU) in the seed region of the guide sequence could be responsible for decreased CRISPR activity [34]. Thus, a more stringent assessment was applied to evaluate the impact of potential transcription ending signal by searching for UUU in the last six bases of the gRNA. UUU was significantly absent in the seed region of functional gRNAs compared with that in nonfunctional gRNAs $(0.8 \%$ versus $8.4 \%, P=8.8 \mathrm{E}-7)$.

\section{Overall nucleotide usage}

Within the 20-nucleotide gRNA sequence, the average counts for adenine were 4.6 and 3.3 for functional and non-functional gRNAs, respectively $(P=9.3 \mathrm{E}-18)$. In contrast, the usage of the other three bases (U, C, or G) was only marginally correlated to CRISPR activity (Table $1 ; P$ values in the range $0.055-0.0019$ ). The preference for adenine in functional gRNAs was not likely a mere reflection of overall preference for GC content as

Table 1 Significant base counts in functional gRNAs

\begin{tabular}{lll}
\hline Mono- or dinucleoside count & Enrichment ratio $^{\mathrm{a}}$ & $P$ value \\
\hline A & 1.39 & $9.3 \mathrm{E}-18$ \\
U & 0.89 & $1.9 \mathrm{E}-03$ \\
G & 0.92 & $6.2 \mathrm{E}-03$ \\
C & 0.95 & $5.5 \mathrm{E}-02$ \\
GG & 0.64 & $2.3 \mathrm{E}-11$ \\
AG & 1.43 & $1.3 \mathrm{E}-09$ \\
CA & 1.38 & $6.7 \mathrm{E}-09$ \\
AC & 1.47 & $1.2 \mathrm{E}-08$ \\
UU & 0.59 & $7.5 \mathrm{E}-08$ \\
UA & 1.84 & $1.1 \mathrm{E}-07$ \\
GC & 0.77 & $3.2 \mathrm{E}-06$ \\
\hline
\end{tabular}

${ }^{\mathrm{a}}$ The enrichment ratio was determined by comparing the average nucleoside counts of functional gRNAs to that of non-functional gRNAs. ${ }^{\text {b }}$ The $P$ values were calculated with Student's t-test the uracil count was even lower in functional gRNAs than in non-functional ones (4.0 versus 4.4). Overall usage of dinucleosides and trinucleosides was also examined and is summarized in Table 1 and Table S1 in Additional file 1 , respectively. The most significant dinucleoside was GG $(P=2.3 \mathrm{E}-11)$ and the most significant trinucleoside was GGG $(P=4.9 \mathrm{E}-13)$. Both GG and GGG were significantly depleted in functional gRNAs, with enrichment ratios of 0.64 and 0.39 , respectively.

\section{Position-specific nucleotide composition}

Base usage at individual positions was summarized and compared between functional and non-functional gRNAs (Table S2 in Additional file 1). Consistent with previous findings [21], there was a strong bias against $U$ and $C$ at the end of functional gRNAs. Interestingly, a $\mathrm{U}$ or $\mathrm{C}$ at the end of the gRNA has a strong propensity to pair with AAG at positions 51-53 of the sgRNA, resulting in an extended stem-loop secondary structure (Fig. 1a). Thus, the bias against $\mathrm{U}$ and $\mathrm{C}$ here was consistent with the structural analysis results, indicating the importance of free accessibility of the seed region for target recognition.

\section{Combining heterogeneous features for genome-wide pre- diction of sgRNA activity}

Identified significant sgRNA features, including both structural and sequence features described above (summarized in Table S3 in Additional file 1), were combined and modeled in a support vector machine (SVM) framework. With these features, a computational algorithm was developed to predict the CRISPR activities. Similar to the sample selection strategy adopted in feature analysis, the most potent sgRNAs (top $20 \%$ in ranking) and the least potent sgRNAs (bottom $20 \%$ ) were included in the SVM training process. The performance of the SVM model was validated by receiver operating characteristic (ROC) curve analysis. To reduce potential risk of overtraining, tenfold cross-validation was performed in this ROC analysis. As shown in Fig. 3a, the area under the curve (AUC) was 0.92 for the SVM model. To further evaluate potential gene-specific bias in model performance, leave-one-geneout cross-validation was performed. Specifically, experimental data from eight of the nine genes were used to train an SVM model while the data from the remaining gene were used for model testing in each iteration of the cross-validation process. The result of this gene-based cross-validation was similar to that of tenfold crossvalidation, with an AUC of 0.91. Thus, our SVM prediction model could be used to differentiate functional sgRNAs from non-functional ones. In summary, crossvalidation analysis indicated that our SVM model, which integrated both structural and sequence features, had robust performance at predicting sgRNA activities. 


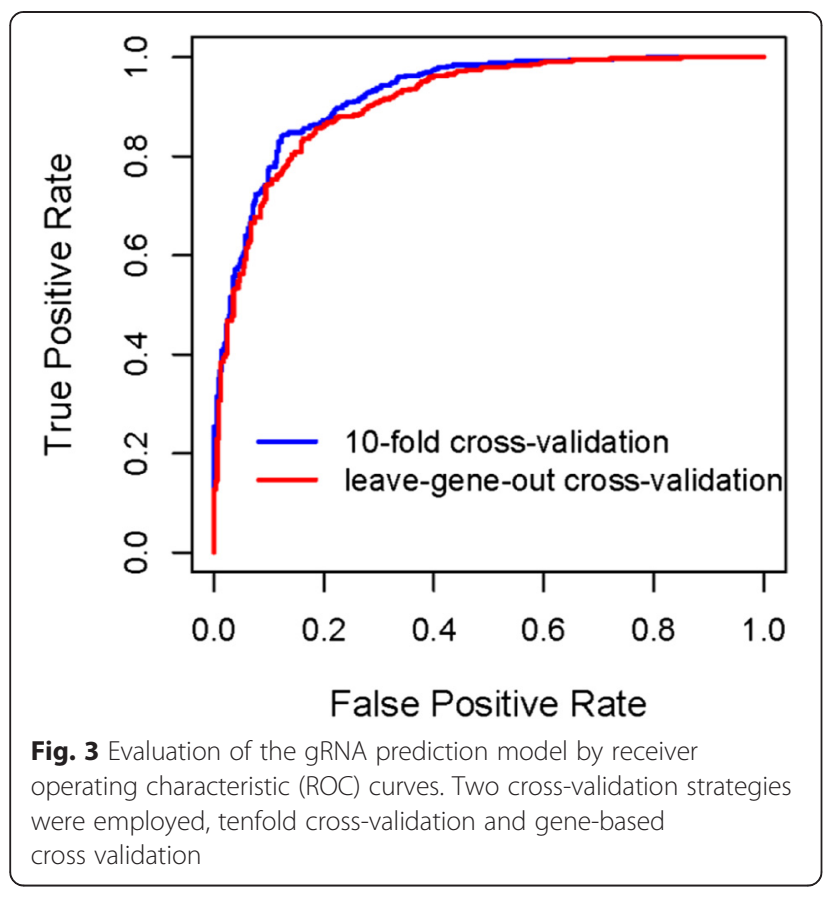

The SVM model was used to select functionally active sgRNAs for all known genes in the human and mouse genomes. To significantly speed up the selection process, a set of pre-filters were implemented to first quickly eliminate unpromising sgRNA candidates before evaluation by the SVM model. These pre-filters are summarized in Table 2. With these pre-filters, about $85 \%$ of non-functional sgRNAs were excluded while about $60 \%$ of functional sgRNAs were retained for further evaluation. Thus, application of the prefilters led to a drastic reduction of non-functional sgRNAs while accompanied by only a moderate increase in the false negative rate. By implementing these pre-filters before SVM modeling, a modified prediction model was constructed for genome-wide sgRNA design based on pre-screened training data.

Table 2 gRNA feature filters that were applied before the SVM modeling process

\begin{tabular}{llc}
\hline Filtered features & Excluded value & $\begin{array}{c}\text { Enrichment ratio for } \\
\text { non-functional gRNA }\end{array}$ \\
\hline gRNA folding $(\Delta \mathrm{G})$ & $<-8 \mathrm{kcal} / \mathrm{mol}$ & 15.8 \\
Duplex binding $(\Delta \mathrm{G})$ & $<-22 \mathrm{kcal} / \mathrm{mol}$ & 3.5 \\
GC content & $>80 \%$ & 30.7 \\
UUU in the seed region & True & 10.5 \\
Repetitive bases & True & 4.2 \\
Position 19 & $\mathrm{U}$ & 2.6 \\
Position 20 & $\mathrm{C}$ or U & 2.5 \\
\hline
\end{tabular}

Free energy $(\Delta \mathrm{G})$ was calculated by RNAfold for gRNA self-folding and by the nearest neighbor method for binding stability of gRNA-target duplex
The general applicability of the SVM model, which we named WU-CRISPR, was evaluated using an independent experimental dataset generated by Chari et al. [28]. In the Chari study, the knockout activities of 279 sgRNAs were determined experimentally by high-throughput sequencing and used to train a novel sgRNA design algorithm, sgRNAScorer. In our analysis, the activities of these sgRNAs were predicted with WU-CRISPR and correlated to experimental data. Furthermore, the performance of three other design tools, sgRNA Designer [21], SSC [27], and sgRNAScorer [28], were also evaluated using the Chari dataset. The Chari dataset was independent from WU-CRISPR, sgRNA Designer, and SSC, but was used to train sgRNAScorer. Thus, tenfold cross-validation results from sgRNAScorer (as presented in the Chari study) were included in our comparative analysis to reduce potential training bias. For each algorithm, top ranking sgRNAs were selected and their knockout activities were checked against the experimental results. Precision-recall curve analysis was performed to evaluate the prediction accuracy. Precision-recall curves are commonly used to evaluate prediction precision (proportion of true positives among all predicted positives) in relation to the recall rate (proportion of true positives among all positive samples). As shown in Fig. 4, all four algorithms performed significantly better than random selection (113 functional sgRNAs among 279 tested sgRNAs, or $40.5 \%$ precision background). Among these algorithms, WU-CRISPR had the best performance at selecting functional sgRNAs. Specifically, all ten sgRNAs with the highest prediction scores by WU-CRISPR were experimentally confirmed to have high knockout activities. Similarly, among all 50 sgRNAs

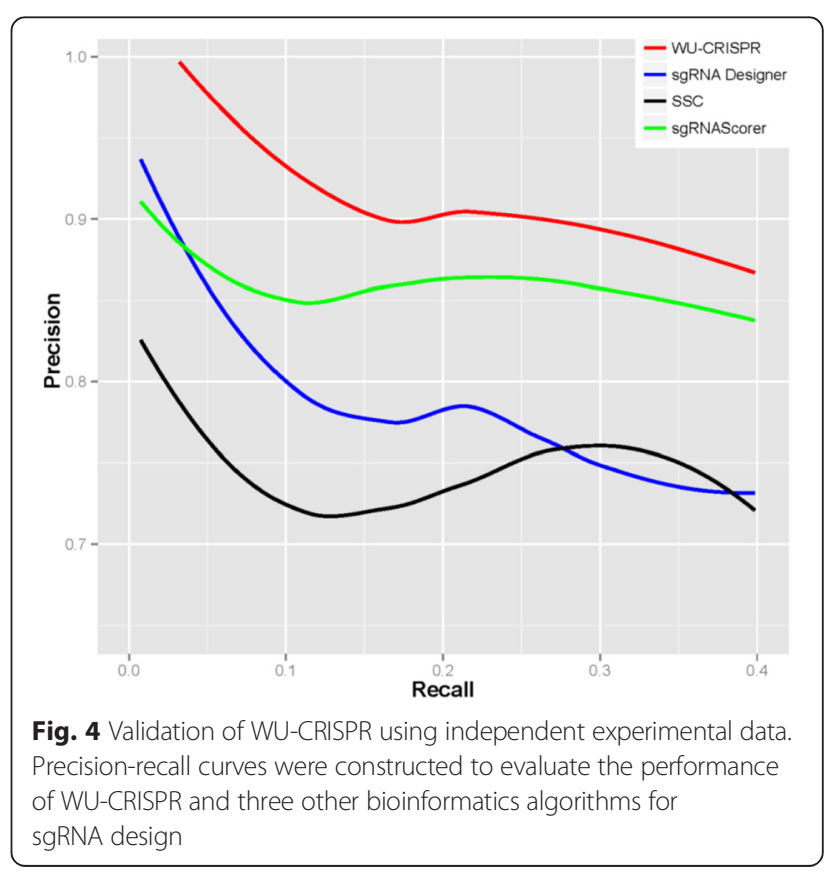


with the highest prediction scores by WU-CRISPR, $88 \%$ were experimentally validated for their high knockout activities.

Besides knockout efficacy, targeting specificity was also considered in the design pipeline. Targeting specificity of sgRNAs has been considered in previously published algorithms. However, existing algorithms search for potential off-target sites in the entire genome space. As the genome contains billions of nucleotides, sgRNA offtargeting is an unavoidable problem when all genomic regions are considered. Recent studies indicate that small-scale genomic alterations (insertions or deletions of less than 20 nucleotides) induced by sgRNA had little functional consequence if the sites are within noncoding regions $[21,22]$. Therefore, we decided to focus our off-targeting analysis exclusively on exon regions, including sequences from both protein-coding genes and other types of genes such as microRNAs and long noncoding RNAs. In this way, more stringent offtarget filters could be implemented since a much smaller sequence space (compared with the entire genome space) was searched.

Each gRNA candidate was compared with all known exon sequences in the genome. Recent experimental studies revealed that the $3^{\prime}$ end seed region of the gRNA is more relevant to off-targeting than the nucleotides residing in the $5^{\prime}$ end. Thus, a more stringent filter is applied to this PAM-proximal seed region. In our algorithm, a gRNA candidate was excluded if its seed sequence ( $3^{\prime}$ end 13 nucleotides) was found in any other unintended exon sequence preceding the PAM domain (NGG or NAG). Furthermore, BLAST sequence alignment was performed to identify and exclude 20-nucleotide gRNA candidates that have over $85 \%$ similarity to any unintended sequence in the design space.

Using the established bioinformatics design pipeline to screen for both CRISPR efficacy and specificity, gRNA sequences were designed to target most known protein-coding genes in the genomes, including 18,635 human and 20,354 mouse genes, respectively. These gRNA sequences are freely accessible via a web server, WU-CRISPR [35]. In addition, a custom design interface was established for gRNA selection based on userprovided sequences.

\section{Discussion}

In a short period of time, the CRISPR/Cas9 system has quickly become a major tool for editing of mammalian genomes. However, the rules governing the efficacy of CRISPR have not been well characterized and most users still design CRISPR assays by trial and error. This problem resembles a similar efficacy issue for RNA interference studies ten years ago when the characteristics of functional siRNAs had not yet been well defined. As a result of significant advances in identifying the features that are characteristic of functional siRNAs, highly active siRNAs can be readily designed with bioinformatics tools, leading to drastic savings in experimental resources. In the current study, we focused on identifying significant features that can be used to predict highly active sgRNAs. Specifically, we reanalyzed a public CRISPR dataset and discovered many novel features that are characteristic of functional sgRNAs. Previously, we and others have shown that both sequence and structural features of the siRNAs are important for RNA interference knockdown activities [30]. Similarly, the knockout activities of CRISPR/Cas9 are also correlated to both sequence and structural features of the sgRNAs. By incorporating heterogeneous features in a prediction model, we have developed an improved bioinformatics design tool and implemented a web server, WU-CRISPR, for genomewide selection of gRNAs for the CRISPR/Cas9 system. The availability of this program may help to improve the efficiency of CRISPR assay design, leading to significant savings in experimental resources at subsequent screening stages.

\section{Materials and methods}

\section{Retrieval of public data for algorithm training}

All gene sequences, including both exon and intron sequences, were downloaded from the UCSC Genome Browser [36]. Index files mapping transcript accessions to NCBI Gene IDs were downloaded from the NCBI ftp site [37]. The Doench dataset for functional sgRNA screening was downloaded from the journal's website [21]. In this published study, functional activities of 1841 sgRNAs were determined by flow cytometry. The Doench dataset was reanalyzed to identify novel features that are correlated to sgRNA efficacy.

\section{Computational tools and data analysis}

LIBSVM was used to build computational models for sgRNA design [38]. For SVM analysis, a radial basis function (RBF) was used for kernel transformation. Optimization of the RBF kernel parameters was done with grid search and cross-validation according to the recommended protocol by LIBSVM. RNA secondary structures and folding energies were calculated with RNAfold [33]. The predicted structures were examined at single-base resolution to determine whether individual nucleotides were base-paired or unpaired in the RNA structures. Statistical computing was performed with the $\mathrm{R}$ package [39]. Statistical significance ( $P$ value) for individual features was calculated by comparing functional and non-functional gRNAs with Student's t-test or $\chi^{2}$ test. 


\section{Validation of WU-CRISPR with independent experimental data}

The Chari dataset [28] was employed to independently evaluate the performance of WU-CRISPR. In the Chari study, the knockout activities of 279 sgRNAs designed for Cas9 (from Streptococcus pyogenes) were determined experimentally by high-throughput sequencing and used to train an sgRNA design algorithm, sgRNAScorer. In our comparative analysis, the Chari dataset was used to compare the performance of WU-CRISPR with three other public algorithms, including sgRNA Designer [21], SSC [27], and sgRNAScorer [28]. Tenfold cross-validation results from sgRNAScorer were previously presented in the Chari study and included in this comparative analysis. The sgRNA Designer program was downloaded at [40]; the SSC program was downloaded at [41]. These standalone tools were used to predict sgRNA activities, and the prediction results were then compared to experimental data. Precision-recall curve analysis was done for algorithm comparison in $\mathrm{R}$ using the ROCR package, and plotted using the ggplot and stat_smooth functions in the ggplot2 package.

\section{Data availability}

The web server and stand-alone software package for gRNA design using the new design algorithm are distributed under the GNU General Public License and are available at [35]. All sequencing data from the Doench study [21] and Chari study [28] can be retrieved from the NCBI Sequence Read Archive (accessions [SRA:SRP 048540] and [SRA:SRP045596], respectively).

\section{Additional file}

Additional file 1: Supplementary Tables S1-S3. (PDF 285 kb)

\section{Abbreviations}

AUC: area under the curve; CRISPR: clustered regularly interspaced palindromic repeat; crRNA: CRISPR RNA; gRNA: guide RNA; PAM: protospacer adjacent motif; RBF: radial basis function; ROC: receiver operating characteristic; sgRNA: single guide RNA; siRNA: small interfering RNA; SVM: support vector machine; tracrRNA: trans-activating crRNA.

\section{Competing interests}

The authors declare that they have no competing interests.

\section{Authors' contributions}

XW designed the study. NW, WL, and XW carried out research. XW and NW wrote the manuscript. All authors read and approved the final manuscript.

\section{Acknowledgements}

We thank Matt Narens for technical assistance. We thank Raj Chari for providing cross-validation data as presented in [28]. This work was supported by the National Institutes of Health [R01GM089784 to X.W.].

Received: 15 July 2015 Accepted: 18 September 2015

Published online: 02 November 2015

\section{References}

1. Doudna JA, Charpentier E. Genome editing. The new frontier of genome engineering with CRISPR-Cas9. Science. 2014;346:1258096.

2. Brouns SJ, Jore MM, Lundgren M, Westra ER, Slijkhuis RJ, Snijders AP, et al. Small CRISPR RNAs guide antiviral defense in prokaryotes. Science. 2008;321:960-4.

3. Marraffini LA, Sontheimer EJ. CRISPR interference limits horizontal gene transfer in staphylococci by targeting DNA. Science. 2008;322:1843-5.

4. Barrangou R, Fremaux C, Deveau H, Richards M, Boyaval P, Moineau S, et al. CRISPR provides acquired resistance against viruses in prokaryotes. Science. 2007;315:1709-12.

5. Jinek M, East A, Cheng A, Lin S, Ma E, Doudna J. RNA-programmed genome editing in human cells. Elife. 2013;2:e00471

6. Cong L, Ran FA, Cox D, Lin S, Barretto R, Habib N, et al. Multiplex genome engineering using CRISPR/Cas systems. Science. 2013;339:819-23.

7. Mali P, Yang L, Esvelt KM, Aach J, Guell M, DiCarlo JE, et al. RNA-guided human genome engineering via Cas9. Science. 2013;339:823-6.

8. Shalem O, Sanjana NE, Hartenian E, Shi X, Scott DA, Mikkelsen TS, et al. Genome-scale CRISPR-Cas9 knockout screening in human cells. Science. 2014;343:84-7

9. Koike-Yusa H, Li Y, Tan EP, Velasco-Herrera Mdel C, Yusa K. Genome-wide recessive genetic screening in mammalian cells with a lentiviral CRISPR-guide RNA library. Nat Biotechnol. 2014:32:267-73.

10. Hwang WY, Fu Y, Reyon D, Maeder ML, Tsai SQ, Sander JD, et al. Efficient genome editing in zebrafish using a CRISPR-Cas system. Nat Biotechnol. 2013;31:227-9

11. Tsai SQ, lafrate AJ, Joung JK. Genome editing: a tool for research and therapy: towards a functional understanding of variants for molecular diagnostics using genome editing. Nat Med. 2014;20:1103-4.

12. Deltcheva E, Chylinski K, Sharma CM, Gonzales K, Chao Y, Pirzada ZA, et al. CRISPR RNA maturation by trans-encoded small RNA and host factor RNase III. Nature. 2011:471:602-7.

13. Jinek M, Chylinski K, Fonfara I, Hauer M, Doudna JA, Charpentier E. A programmable dual-RNA-guided DNA endonuclease in adaptive bacterial immunity. Science. 2012;337:816-21.

14. Hsu PD, Scott DA, Weinstein JA, Ran FA, Konermann S, Agarwala V, et al. DNA targeting specificity of RNA-guided Cas9 nucleases. Nat Biotechnol. 2013:31:827-32.

15. Heigwer F, Kerr G, Boutros M. E-CRISP: fast CRISPR target site identification. Nat Methods. 2014:11:122-3.

16. Montague TG, Cruz JM, Gagnon JA, Church GM, Valen E. CHOPCHOP: a CRISPR/Cas9 and TALEN web tool for genome editing. Nucleic Acids Res. 2014:42:W401-7

17. Naito Y, Hino K, Bono H, Ui-Tei K. CRISPRdirect: software for designing CRISPR/ Cas quide RNA with reduced off-target sites. Bioinformatics. 2015;31:1120-3.

18. Pattanayak V, Lin S, Guilinger JP, Ma E, Doudna JA, Liu DR. High-throughput profiling of off-target DNA cleavage reveals RNA-programmed Cas9 nuclease specificity. Nat Biotechnol. 2013;31:839-43.

19. Tsai SQ, Zheng Z, Nguyen NT, Liebers M, Topkar W, Thapar V, et al. GUIDE-seq enables genome-wide profiling of off-target cleavage by CRISPR-Cas nucleases. Nat Biotechnol. 2015;33:187-97.

20. Kim D, Bae S, Park J, Kim E, Kim S, Yu HR, et al. Digenome-seq genome-wide profiling of CRISPR-Cas9 off-target effects in human cells. Nat Methods. 2015;12:237-43.

21. Doench JG, Hartenian E, Graham DB, Tothova Z, Hegde M, Smith I, et al. Rational design of highly active sgRNAs for CRISPR-Cas9-mediated gene inactivation. Nat Biotechnol. 2014:32:1262-7.

22. Ho TT, Zhou N, Huang J, Koirala P, Xu M, Fung R, et al. Targeting non-coding RNAs with the CRISPR/Cas9 system in human cell lines. Nucleic Acids Res. 2015:43:e17.

23. Ran FA, Hsu PD, Lin CY, Gootenberg JS, Konermann S, Trevino AE, et al. Double nicking by RNA-guided CRISPR Cas9 for enhanced genome editing specificity. Cell. 2013;154:1380-9.

24. Mali P, Aach J, Stranges PB, Esvelt KM, Moosburner M, Kosuri S, et al. CAS9 transcriptional activators for target specificity screening and paired nickases for cooperative genome engineering. Nat Biotechnol. 2013:31:833-8.

25. Wang T, Wei JJ, Sabatini DM, Lander ES. Genetic screens in human cells using the CRISPR-Cas9 system. Science. 2014;343:80-4.

26. Gagnon JA, Valen E, Thyme SB, Huang P, Ahkmetova L, Pauli A, et al. Efficient mutagenesis by Cas9 protein-mediated oligonucleotide insertion and large-scale assessment of single-guide RNAs. PLoS One. 2014;9:e98186. 
27. Xu H, Xiao T, Chen CH, Li W, Meyer CA, Wu Q, et al. Sequence determinants of improved CRISPR sgRNA design. Genome Res. 2015;25:1147-57.

28. Chari R, Mali P, Moosburner M, Church GM. Unraveling CRISPR-Cas9 genome engineering parameters via a library-on-library approach. Nat Methods. 2015;12:823-6.

29. Zhu LJ, Holmes BR, Aronin N, Brodsky MH. CRISPRseek: a bioconductor package to identify target-specific guide RNAs for CRISPR-Cas9 genomeediting systems. PLoS One. 2014;9:e108424.

30. Wang X, Wang X, Varma RK, Beauchamp L, Magdaleno S, Sendera TJ. Selection of hyperfunctional siRNAs with improved potency and specificity. Nucleic Acids Res. 2009;37:e152

31. Long $D$, Lee $R$, Williams $P$, Chan $C Y$, Ambros $V$, Ding Y. Potent effect of target structure on microRNA function. Nat Struct Mol Biol. 2007;14:287-94.

32. Robins H, Li Y, Padgett RW. Incorporating structure to predict microRNA targets. Proc Natl Acad Sci U S A. 2005;102:4006-9.

33. Hofacker IL. Vienna RNA, secondary structure server. Nucleic Acids Res. 2003;31:3429-31

34. Wu X, Scott DA, Kriz AJ, Chiu AC, Hsu PD, Dadon DB, et al. Genome-wide binding of the CRISPR endonuclease Cas9 in mammalian cells. Nat Biotechnol. 2014;32:670-6.

35. WU-CRISPR. http://crispr.wustl.edu.

36. Karolchik D, Barber GP, Casper J, Clawson H, Cline MS, Diekhans M, et al. The UCSC Genome Browser database: 2014 update. Nucleic Acids Res. 2014;42:D764-70.

37. Resource Coordinators NCBI. Database resources of the National Center for Biotechnology Information. Nucleic Acids Res. 2015;43:D6-17.

38. LIBSVM. http://www.csie.ntu.edu.tw/ cjlin/libsvm/.

39. R Project. http://www.r-project.org/.

40. sgRNA Designer. http://www.broadinstitute.org/rnai/public/analysis-tools/ sgrna-design.

41. SSC. http://sourceforge.net/projects/spacerscoringcrispr/.

\section{Submit your next manuscript to BioMed Central and take full advantage of:}

- Convenient online submission

- Thorough peer review

- No space constraints or color figure charges

- Immediate publication on acceptance

- Inclusion in PubMed, CAS, Scopus and Google Scholar

- Research which is freely available for redistribution 